\title{
Inmediatez y veracidad de los hechos, un compromiso desdibujado desde el periodismo
}

Immediacy and veracity of the facts, a commitment blurred from journalism

Intermediação e veracidade dos fatos, compromisso obscurecido pelo jornalismo

\section{Wilson Benjamín LEÓN VALLE}

Universidad Estatal Península de Santa Elena (Ecuador)

wleon@upse.edu.ec

Gabriela Lourdes VÉLEZ BERMELLO

Universidad Estatal Península de Santa Elena (Ecuador)

gabriela-8906@hotmail.com

Chasqui. Revista Latinoamericana de Comunicación

N. ${ }^{\circ}$ 148, diciembre 2021 - marzo 2022 (Sección Diálogo de saberes, pp. 295-312)

ISSN 1390-1079 / e-ISSN 1390-924X

Ecuador: CIESPAL

Recibido: 28-o9-2021 / Aprobado: 12-11-2021 


\title{
Resumen
}

Con el incremento exponencial de información en Internet, los medios tradicionales han sufrido convergencias cohesionando el periodismo con el uso de redes sociales, enmarcado en la inmediatez por compartir nuevos contenidos, ocasionando que se agrave el proceso de construcción de la noticia y que la verificación de los hechos vaya quedando en el olvido. La presente investigación tiene el objetivo de identificar cómo se desarrolla el ejercicio periodístico en el la provincia de Santa Elena- Ecuador, a través la técnica de grupo focal y encuestas a la población, se ha evidenciado la necesidad de replantear bases procedimentales en el periodismo, puesto que la ciudadanía está ávida de certezas inmediatas, de lo contrario el ciudadano común construye su propia verdad con datos generados desde la desinformación.

Palabras clave: verificación de datos; información mediática; tratamiento de noticias; compromiso ético; ética.

\begin{abstract}
With the exponential increase in information on the Internet, the traditional media have converged, uniting journalism with the use of social networks, framed in the immediacy of sharing new content, causing the process of news construction to worsen and the verification of the facts are being forgotten. The present investigation has the objective of identifying how journalistic practice is carried out in the province of Santa Elena-Ecuador, through the focus group technique and population surveys, the need to rethink procedural bases in journalism has been evidenced, since the citizenry is hungry for immediate certainties, otherwise the common citizen constructs his own truth with data generated from disinformation.
\end{abstract}

Keywords: data verification; media information; news treatment; ethical commitment; ethics.

\section{Palavras-chave}

Com o aumento exponencial das informações na Internet, as mídias tradicionais convergiram, unindo o jornalismo ao uso das redes sociais, enquadradas no imediatismo do compartilhamento de novos conteúdos, agravando o processo de construção de notícias e a verificação de os fatos estão sendo esquecidos. Esta pesquisa tem como objetivo identificar como a prática jornalística é realizada na província de Santa Elena-Equador, através da técnica de grupos focais e pesquisas populacionais, foi evidenciada a necessidade de repensar as bases processuais no jornalismo, como os cidadãos têm fome de certezas imediatas, caso contrário, o cidadão comum constrói sua própria verdade com dados gerados a partir da desinformação.

Palavras chave: verificação de dados; informações de mídia; tratamento de notícias; compromisso ético; ética. 


\section{Introducción}

Los medios de comunicación están sometidos a la continua crítica, por la forma de abordar ciertos hechos noticiosos, que evidencian la práctica de valores éticos en el ejercicio de la profesión; en este contexto, Eco (2003), escritor ligado al periodismo manifiesta en su artículo: "La labor crítica del periodismo", que esto no es más que el fiel reflejo de los diferentes atropellos cometidos por los medios de comunicación tradicional y digitales a la sociedad (Eco, Guerra y Web 2.0, descripción y esperanza frente a los medios digitales, 2016).

En materia de periodismo, existen investigaciones respecto al tratamiento de la noticia y la inmediatez, por ejemplo, Alsina (1989) se enfoca específicamente en la radio y menciona cómo este medio induce a las personas y proyecta pensamientos prefabricados por quienes están detrás de los micrófonos, teniendo como premisa que la radio incentiva a la imaginación de los oyentes.

De hecho, autores como Borges (1998) vaticinaba que la objetividad; que en teoría se enseña en las aulas, no era más que una utopía, algo que en la práctica se difuminaba entre los intereses del medio y de ciertos periodistas. Este investigador, incluso asume que dicha objetividad es un mito persistente y que el origen por el cual surgió el periodismo, se encamina actualmente lejos de la imparcialidad y la honestidad.

Años después, Canavilhas e Ivars (2012) coinciden con Borgues, pero hace un alcance hacia el periodismo 2.0, reafirmando la falta de credibilidad, con ello se expone que las fuentes periodísticas tradicionales son fundamentales para conformar una información, pero que poco a poco nacen fuentes alternativas, como las que se encuentran en la web y éstas necesitan tener un proceso riguroso de verificación para ser parte de una noticia. El incremento de más investigadores preocupados por el destino de los contenidos que se emiten en medios es exponencial (Canavilhas J. , 2012).

El libro: "Los riesgos del periodismo en tiempos de redes" De Alzaga (2012) revela los vicios frecuentes en el periodismo cuando se utilizan las redes sociales como fuentes de información, sin embargo, recalca que dentro de las buenas prácticas existen periodistas responsables. Se debe recordar que antes de Internet, el medio de comunicación más inmediato era la radio y que en la actualidad ambas se manifiestan casi de forma instantánea a los hechos. Romero (2012) manifiesta que existe un lazo entre radio y redes sociales, pues ambas toman protagonismos, más aún si una misma empresa de comunicación tiene una fanpage de la emisora radial. Estas mismas conjunciones se suscitan en los otros medios tradicionales (De Alzaga, y otros, 2012).

Volver a las fuentes confiables, chequear la información brindada, contrastar los hechos, completar con datos y cifras, son algunos de los planeamientos que sugiere Aller (2012) en su artículo: La verdad periodística y las fuentes de información, esto en una búsqueda desesperada por frenar la propagación de noticias sin fundamentos profesionales (Aller, 2012). 
El Manual de periodismo independiente de Potter brinda una orientación apropiado de tratamiento informativo para la construcción de noticias (Potter, 2006). En el territorio, estrictamente en la provincia de Santa Elena, existen trabajos como El tratamiento de la información desde la perspectiva ética para mejorar la calidad noticiosa, un Estudio de caso: radiodifusoras Amor y Récord del cantón La Libertad, uno de los pocos estudios realizados en la misma jurisdicción y coincide con una de las variables de la presente investigación (Orrala \& Vera, 2014).

El artículo: "La prudencia en la inmediatez del acto informativo" (Rojas y Tomás, 2012) expone las contraposiciones conceptuales entre inmediatez y prudencia, también revela las discrepancias éticas a la que está expuesto el periodista al momento de enfrentar hechos noticiosos que deben informarse de inmediato, a la vez plantea una posible conciliación entre los dos términos para mejorar la práctica periodística. Esta publicación se vincula notablemente con la hipótesis de este trabajo y en la correlación de las variables de la presente en la investigación (Rojas \& R, 2012 ).

\section{Marco teórico}

Existen amplios estudios del proceso en la elaboración de contenidos noticiosos, Sigal (1973), hace una radiografía del reporterismo, demostrando las bases en las cuales se construye la información y de acuerdo a las fechas. Autores como Fishman (1980), Tuchman (1983) y Wolf (1997), han contribuido con los diversos conceptos y transformaciones del manejo de contenidos, además muestran críticas al ejercicio de la profesión, poniendo en duda las objetividades al momento de informar, producto de la inmediatez en un mundo globalizado, en pleno auge de la sociedad del conocimiento y la información.

La sobre exposición a información en este mundo globalizado, puede producir en la ciudadanía infoxicación, término utilizado por Cornella, que hace referencia a la sobreabundancia de información, la credibilidad se ve relegada por los posts, que se suben segundo a segundo en redes sociales o en diferentes plataformas, creando con ello la desinformación y cayendo en construcciones de realidades con poco asidero a la argumentación (Cornella, 2000).

La participación en la generación de contenidos en redes va desde cualquier persona que tenga un mediano acceso a Internet, trayendo con ellos la denominada legiones de idiotas, como la denominó (Eco, 2016), quien no ha dudado en sentenciar así a quienes postean cualquier cosa en redes, sin escrúpulos, responsabilidad y sin verificar fuente.

En estecontexto se conoceque el periodismo en surecolección deinformación tiene una ardua tarea, sobre todo, en la verificación de los hechos, por ello, Salaverría quien estudia a fondo el periodismo digital y su convergencia, afirma que esta actividad está en un constante cambio, por lo que al comunicador no le 
queda más que ajustarse al rigor de la verificación de las fuentes, evitando caer en lo que el ciudadano común suele postear, noticias falsas (Salavarría, 2010).

Un estudio realizado por la Universidad del Nordeste en Boston (Estados Unidos), devela que los bulos o noticias falsas tienen una gran trascendencia, al igual que un hecho o sucesos debidamente investigado y contrastado, en redes sociales como Facebook (Castillo, 2017). Dicho alcance puede generar conmoción o caos, por lo cual autoridades gubernamentales se han visto en la necesidad de emitir llamados de atención ante esta práctica.

Un factor primordial en el mundo digital en el ámbito comunicacional es, la inmediatez, que permite la actualización constante de la información llegando con ello a superar tanto a la radio como a la televisión (Flora Marín, José Ignacio Armentia, José Caminos, 2011). Este factor resulta por ende un diferencial del modelo económico, productivo y profesional del XX, considerando que lo que ayer resultaba útil hoy es obsoleto (García, 2010). Se podría identificar, entonces, que medios tradicionales como la radio se ven en la necesidad de ajustar sus contenidos a un mundo de inmediatez y no aportando en la contrastación y, sobre todo, verificación de información.

(...) es cierto que las propias ediciones digitales, tanto de los periódicos como de los medios audiovisuales, se han convertido en competidores directos de las agencias de noticias, ya que publican las noticias incluso con más rapidez. Y esto también ha servido de acicate para ganar en celeridad. (Monfort, 2013)

Garavito \& Ramírez, consideran que este aspecto de la comunicación en el ámbito digital requiere de un proceso de filtración tomando en cuenta que en estos tiempos cualquier individuo pude hacer del papel de informador, pudiendo en muchos casos encontrarse con noticias erróneas o falsas publicadas en la red e incluso caer en las manos de comunicadores de algún medio tradicional que replique los contenidos, sin ninguna verificación (Aguilar-Garavito \& Ramírez, 2015).

Por lo tanto, se ha de valorar de forma preferencial la calidad a la inmediatez. Por ello, ha de estimarse al comunicador actual como un competidor más en busca de información, teniendo contrincantes como el ciudadano común, cuyo Smartphone lo pone al tanto no solo del quehacer público, también tienen la oportunidad de generar contenidos.

Se evidencia de esta manera a un nuevo tipo de audiencia, la cual no precisa esperar el inicio del noticiero radial, sino que puede hacerlo a la hora y en el lugar que le convenga mediante su dispositivo móvil (Enriquez \& Casas, 2013).

Para Rosique \& Barranquero, la inmediatez es una aliada del marketing actual, debiéndose tener cierto cuidado con la misma al momento de informar puesto que lo que se pone en juego es la credibilidad, la cual constituye un valor fundamental dentro y fuera de los medios de comunicación. Una primicia puede en algunos casos resultar una ventaja contra los demás medios, pero a su vez 
puede convertirse en un arma de doble filo en contra del mismo si aquello que se informó termina generando confusión o pánico, dependiendo de la magnitud del caso (Rosique-Cedillo \& Barranquero-Carretero, 2015). .

Ruiz, se refiere al honor y al buen nombre como un derecho sobre el cual no se debe ser negligente ni cobarde, siendo, por lo tanto, un defecto el dar las cosas por hechas sin tener una información veraz, verificada, oportuna y contextualizada denigrando con esta acción el buen nombre de una persona (Ruiz, 2014).

\section{Metodología}

La investigación pretende explicar la relación que guarda la inmediatez en el tratamiento de la información de los hechos noticiosos, pretende hacerlo desde el punto de vista de los actores del proceso, los comunicadores y periodistas en la provincia de Santa Elena. Desde esta perspectiva, la investigación tiene un enfoque mixto; cuali-cuantitativo, debido a que intenta fundamentar la forma que se realiza el proceso informativo (Hernandez Sampieri, 2016).

Las técnicas para la recolección de datos utilizada es el grupo focal, dirigida a los profesionales que trabajan en los medios de comunicación, esto es, con el fin de identificar el proceso utilizado para darle tratamiento a la noticia, esta técnica permite la indagación e interpretación de fenómenos que no pueden ser tratados bajo la observación científica (Hamui Sutton \& Varela Ruiz, 2012). Adicionalmente, se procedió a realizar una encuesta a la población, para conocer los criterios respecto como la población asume la inmediatez de la información.

Para determinar el universo, para la selección del grupo focal, se tomó en cuenta las emisoras que tienen frecuencia como matriz en la provincia de Santa Elena, en cuya parrilla conste noticieros de producción local, esto no incluye las retransmisiones de informativos que se producen fuera la jurisdicción mencionada. Según el portal de la Agencia de Regulación y Control de las Telecomunicaciones (ARCOTEL). Para la tabulación de los resultados se utilizó el programa de análisis cualitativo Atlas.ti 8.

En cuanto a la encuesta, se identificó una población de 229.772 mayor de 18 años inscrita en el padrón electoral provincial, cuya muestra es de 399 habitantes. La muestra se estratificó en los tres cantones; Salinas 87, La Libertad 124, Santa Elena 188. El criterio de selección, fue un muestreo aleatorio simple, con el propósito de establecer una estadística que permita identificar e interpretar el comportamiento de la población en sus prácticas cotidianas.

\section{Resultados}

El grupo focal está compuesto por dos periodistas que realizan su trabajo desde la cabina de la estación radial y dos reporteros de campo, quienes se movilizan por la provincia en búsqueda de noticias: 
Tabla 1. Listado de Periodistas participantes en el grupo focal

\begin{tabular}{|l|l|l|}
\hline Nombres y apellidos & Cargo en la radio & Experiencia periodística \\
\hline Joffre Lino de la Cruz & $\begin{array}{l}\text { Locutor de cabina y } \\
\text { entrevistador. }\end{array}$ & $\begin{array}{l}\text { 23 años como periodista, redacta para los diarios El Expreso y el } \\
\text { Extra (Grupo GRANASA), cursa el último ciclo de la carrera para } \\
\text { obtener su título de Licenciado en Comunicación Social en la } \\
\text { Universidad Técnica Particular de Loja. }\end{array}$ \\
\hline Priscila del Peso Arias & $\begin{array}{l}\text { Locutora en cabina, } \\
\text { presentadoray } \\
\text { entrevistadora. }\end{array}$ & $\begin{array}{l}\text { Presentadora de noticias y entrevistadora del noticiero en Brisa } \\
\text { TV; administra la FANPAGE de Facebook del periódico El BACAN; } \\
\text { desde hace 10 años redacta para el diario El Universo en las } \\
\text { secciones de Actualidad, Vida y Estilo, Política, Intercultural, País, } \\
\text { Sucesos; colabora como reportera de territorio para Radio City; } \\
\text { Cursa la Maestría en Periodismo Digital en la Universidad de las } \\
\text { Américas. }\end{array}$ \\
\hline Valeria de La Cruz & Reportera de campo & $\begin{array}{l}\text { Licenciada en Comunicación Social, siete años de experiencia en } \\
\text { medios de comunicación, laboró en ESPOL TV como presentadora } \\
\text { de noticias, locutora de programas cristianos y actualmente } \\
\text { dirige también el Periódico el Vocero en todas sus plataformas } \\
\text { (Faecbooky versión impresa) }\end{array}$ \\
\hline David Cruz & Reportero de campo & $\begin{array}{l}\text { Licenciado en Comunicación Social. Inició como reportero de } \\
\text { Radio Pacífico, fue administrador de contenidos en Notipeninsula. } \\
\text { com; Editor de reportajes en ESPOL TV; reportero para Diario } \\
\text { Súper; Administrador de varias FANEPAGE como la del Diario Súper } \\
\text { y Noticias en la Comunidad. }\end{array}$ \\
\hline
\end{tabular}

Elaborado por: Autores

\section{Resultados en análisis cualitativo, Informe de códigos; códigos seleccionados (2)}

\section{El tratamiento del hecho noticioso}

Comentario: por usuario. ¿Cómo se produce el hecho noticioso? 4 Citas:

1:2 El primer paso para el tratamiento del hecho noticioso es la observa (1778:3259) - D 1: Participante 1

El primer paso para el tratamiento del hecho noticioso es la observación, pero no solo el hecho de estar en el sitio para constatar ocularmente la existencia de un acontecimiento, sino de saber ubicar a los vinculados en el hecho y a los testigos, quienes brindarán los datos preliminares antes de iniciar una grabación o trasmitir en vivo. Los periodistas son contundentes en expresar que, ante la inmediatez, no se cumple con el contraste en los noticieros radiales. Dejó claro que únicamente en el contexto radial se obvia el contraste ante la inmediatez, esto no debe ocurrir en televisión ni en los periódicos, en radio se realizan entregas parciales de un hecho noticioso. Los noticieros de las radios provinciales son distintos a sus similares de cobertura nacional, estos últimos no exceden de las dos horas y presentan notas elaboradas y pregrabadas, a más 
de entrevistas. Lo contrario ocurre en Santa Elena, pues son largas jornadas informativas que inician desde las siete de la mañana hasta las doce del día, en el caso particular de Radio Amor, el informativo comienza a las o6hoo y finaliza a las 18hoo. En este mismo contexto Lino destaca que en los medios de comunicación de carácter nacional, los reportajes desde el territorio se realizan en horas determinadas y no duran más de dos minutos, el reportero se ajusta a ese horario, lo que no ocurre en la provincia de Santa Elena. “acá el reportero te llama y dice tiene una noticia y de inmediato y se va de largo

2:2 los reporteros constatan los hechos y envían las grabaciones, pero en... (2399:4023) - D 2: Participante 2

Los reporteros constatan los hechos y envían las grabaciones, pero en muchas ocasiones las notas no están contrastadas debido a que no siempre se encuentran todos los implicados en el lugar del hecho. Cuando los acontecimientos noticiosos implican a personas o instituciones, cuyos representantes no están en el sitio del suceso, obliga al reportero a realizar una segunda entrega del reporte a modo de seguimiento, sin embargo, el mismo sale del contexto de inmediatez ante el hecho inicial. Todos coincidieron en que el contraste no forma parte de un hecho noticioso en el contexto de la inmediatez, se contrasta, pero luego de un tiempo determinado por el reportero, que es el encargado de encontrar a los liados al evento. Por otra parte, realiza una analogía entre la Radio y los medios digitales, y asegura que en ambos se realizan entregas parciales de una noticia, en primera instancia se informa de un hecho importante y se agrega el rótulo "noticia en desarrollo" para dejar claro que más adelante se ampliará la información. Es decir, el contraste se realiza por cuotas y no en contexto de inmediatez. Los periodistas de cabina coinciden en que el proceso del tratamiento del hecho noticioso se realiza en condiciones normales para obtener productos elaborados, y que permiten realizar noticieros estructurados bajo guion. Contrapone este criterio y asegura que la inmediatez existe en la primera salida del reporte de radio, es decir, cuando se realiza la segunda salida para contrastar información difundida minutos antes, sale del contexto de inmediatez y cumple con el proceso de tratamiento de la noticia.

3:2 que mientras se cumpla con el tratamiento correcto de la noticia es di... (1013:1106) - D 3: Participante 3

Que mientras se cumpla con el tratamiento correcto de la noticia es difícil tener inmediatez.

4:2 podría presentar todas las versiones en un solo reporte de radio, pero... (1475:2451) - D 4: Participante 4.

Podría presentar todas las versiones en un solo reporte de radio, pero eso implicaría edición y tiempo para movilizarse a tomar las versiones de todas las partes, pero al mismo tiempo ya no se estaría hablando de un hecho de inmediatez. También destaca que los espacios informativos de las radios no trabajan con guion, además de tener una extensa jornada de noticias lo que provoca de improvisación por parte de los locutores de radio. Otro particular 
que destaca el grupo es que los periodistas radiales de la provincia no manejan libreta de apuntes, los reporteros solo llegan al sitio e inician su transmisión. Pese a la autocrítica, no la denominan como improvisación, sino que la llaman destreza. Los periodistas coinciden en que, si se ejecuta la recopilación de datos previo a iniciar una agravación o un reporte en vivo, pero destacan que hacerlo sin antes tener datos preliminares es un error y que por lo general se comete cuando el periodista inicia en el oficio

\section{La inmediatez de la información}

Comentario: por usuario, ¿Cómo se entiende la inmediatez? 4 Citas:

1:1 plantea tres normas básicas para los reporteros: 1.- Estar en el sitio... (32:1741) - D 1: Participante 1

Plantea tres normas básicas para los reporteros: 1.- Estar en el sitio del hecho noticioso para tener accesibilidad a la información; 2.- Observar para identificar lo novedoso del hecho y darle un buen enfoque a la nota periodística; y 3.Saber contar las historias, debido a que la radio es un medio de comunicación unisensorial, la forma de contar las historias tiene mucho que ver, para que la audiencia pueda elaborar criterios desde su percepción. En cuanto a los dos primeros aspectos, los periodistas determinan que las redes sociales representa un peligro para la información en la práctica cotidiana, se sube la información en el sitio web, esta situación se constituye en una limitante por el acceso directo a las fuentes primarias, por lo tanto, están supeditados a la insuficiente información que los usuarios de las redes sociales les puedan compartir sin verificación de los hechos. Este criterio revela que en la práctica periodística, la verificación juega un rol importante frente a la inmediatez de la información, y al no estar presentes en el sitio, se realiza una breve verificación con fotografía, en este contexto, la aplicación de mensajería WhatsApp se convierte en una herramienta para la trasmisión de hecho noticioso. Lo que implica algún riesgo para el buen ejercicio la profesión. Parten de la premisa que ninguna fuente se pude desechar, todas las fuentes son válidas, lo que hacen es que algunas fuentes sirven para citar y ser parte de la noticia y otras se mantienen el anonimato pero brindan información relevante que otras fuentes obvian a conveniencia, esas fuentes sirven para dar seguimiento silencioso al acontecimiento, el buen periodista no descarta ninguna fuente.

2:1 para los periodistas las fuentes oficiales parecen estar fuera del con... (32:2362) - D 2: Participante 2

para los periodistas las fuentes oficiales parecen estar fuera del contexto de inmediatez, "hasta que se los espera, demoran una eternidad, (...) no necesariamente se dice lo que manifiestan las fuentes, sino que se relata el suceso y esa es la ventaja en la inmediatez, para los profesionales en los medios de comunicación, hasta cierto punto, termina siendo un reto, y también un riesgo. Considera que el reportero de campo está más expuesto a la inmediatez, 
por ello el trabajo en equipo es fundamental, en muchas ocasiones la narración del reportero atropella datos importantes, producto de la aceleración por la inmediatez, pero desde cabina se solicita mayor contextualización y detalles de los hechos, así se mantiene la situación bajo control y una información fluida para los periodistas las fuentes oficiales parecen estar fuera del contexto de inmediatez, "hasta que se los espera, demoran una eternidad, (...) no necesariamente se dice lo que manifiestan las fuentes, sino que se relata el suceso y esa es la ventaja en la inmediatez, para los profesionales en los medios de comunicación, hasta cierto punto, termina siendo un reto, y también un riesgo. Considera que el reportero de campo está más expuesto a la inmediatez, por ello el trabajo en equipo es fundamental, en muchas ocasiones la narración del reportero atropella datos importantes, producto de la aceleración por la inmediatez, pero desde cabina se solicita mayor contextualización y detalles de los hechos, así se mantiene la situación bajo control y una información fluida para los periodistas las fuentes oficiales parecen estar fuera del contexto de inmediatez, "hasta que se los espera, demoran una eternidad, (...) no necesariamente se dice lo que manifiestan las fuentes, sino que se relata el suceso y esa es la ventaja en la inmediatez, para los profesionales en los medios de comunicación, hasta cierto punto, termina siendo un reto, y también un riesgo. Considera que el reportero de campo está más expuesto a la inmediatez, por ello el trabajo en equipo es fundamental, en muchas ocasiones la narración del reportero atropella datos importantes, producto de la aceleración por la inmediatez, pero desde cabina se solicita mayor contextualización y detalles de los hechos, así se mantiene la situación bajo control y una información fluida

3:1 la ventaja que tiene el periodista de radio es que, producto de la inm... (32:976) - D 3: Participante 3

La ventaja que tiene el periodista de radio es que, producto de la inmediatez, tiene a los testigos oculares y a los protagonistas de los hechos en el momento, entonces son ellos mismos los que cuentan su historia y el reportero sirve de enlace entre una entrevista y otra, cosa que no pasa en la televisión o el periódico, en estos casos el periodista es el que cuenta la historia. En hechos de inmediatez, las fuentes primarias son los testigos oculares o familiares de algún afectado, que no pudieran ser voces oficiales para determinar si una persona sin vida en la calzada fue atropellada, asesinada o se autoeliminó, pero informa del contexto, nombres de los protagonistas del hecho, ubicación geográfica, información espacial y temporal y un sinnúmeros de datos que permiten desarrollar la noticia durante una trasmisión, "De no existir fuentes oficiales, los testigos oculares se convierten en fuentes primarias para obtener información

4:1 Frente a la inmediatez, las herramientas tecnológicas son los mejores... (32:1438) - D 4: Participante 4

Frente a la inmediatez, las herramientas tecnológicas son los mejores aliados de los periodistas al momento de enviar un reporte. Para David Cruz, la mejor manera es realizar una grabación de voz en el celular y luego enviarla 
por WhatsApp, con lo cual asegura una buena calidad de audio. La grabación la realiza de forma que pareciera que está transmitiendo en vivo, lo que da al oyente una sensación de inmediatez, sin embargo, para Joffre Lino, es más eficiente la llamada telefónica en vivo, ya que permite una interacción con el reportero y los protagonistas, además ayuda a despejar dudas si fuera necesario fortaleciendo el tratamiento del hecho, lo que no es posible con las grabaciones. Los periodistas que permanecen en la cabina aseguran que no siempre escuchan la nota de voz que envía su reportero por WhatsApp antes de difundirla, actúan con plena confianza en el trabajo periodístico de sus compañeros en el campo, sin embargo, De la Cruz Conforme prefiere enviar un pequeño lead junto con la nota de voz para que en la cabina puedan presentar de mejor manera la noticia y permitan una contextualización previa a la difusión del audio. Pese a las herramientas tecnológicas, el grupo coincide que, ante la inmediatez, el mejor reporte se hace en vivo a través de una llamada de telefonía celular donde el reportero narra lo que observa mientras se moviliza por el sitio y busca a las fuentes

Análisis e interpretación de los resultados de las encuestas:

Tabla. No. 2 Verifica si la información es veraz

\begin{tabular}{|l|l|c|c|c|c|}
\hline \multicolumn{2}{|c|}{} & Frecuencia & Porcentaje & Porcentaje válido & Porcentaje acumulado \\
\hline \multirow{4}{*}{ Válido } & No respondió & 12 & 2,9 & 3,0 & 3,0 \\
\cline { 2 - 6 } & Sí & 264 & 63,5 & 66,2 & 69,2 \\
\cline { 2 - 6 } & No & 123 & 29,6 & 30,8 & 100,0 \\
\cline { 2 - 6 } & Total & 399 & 95,9 & 100,0 & \\
\hline Perdidos & Sistema & 17 & 4,1 & & \\
\hline Total & 416 & 100,0 & & \\
\hline
\end{tabular}

Fuente: población de la provincia de Santa Elena Elaborado por: Autores

Con relación a la pregunta si los encuestados verifican la información que reciben es veraz. El 64 \% responde que no verifica, 27 \% indica que sí verifica.

Tabla. No. 3 Difunde la información como verdadera

\begin{tabular}{|l|l|c|c|c|c|}
\hline \multicolumn{2}{|c|}{} & Frecuencia & Porcentaje & Porcentaje válido & Porcentaje acumulado \\
\hline \multirow{4}{*}{ Válido } & No respondió & 13 & 3,1 & 3,3 & 3,3 \\
\cline { 2 - 6 } & No & 92 & 22,1 & 23,1 & 26,3 \\
\cline { 2 - 6 } & Sí & 294 & 70,7 & 73,7 & 100,0 \\
\cline { 2 - 6 } & Total & 399 & 95,9 & 100,0 & \\
\hline Perdidos & Sistema & 17 & 4,1 & & \\
\hline Total & 416 & 100,0 & & \\
\hline
\end{tabular}

Fuente: población de la provincia de Santa Elena . Elaborado por: Autores 
La pregunta respecto a si difunde la información como verdadera, los encuestados responden en 71 \% sí la difunden, un $22 \%$ que manifiesta que no.

Tabla. No. 4 La información influye en el comportamiento

\begin{tabular}{|l|l|l|l|l|l|}
\hline & & Frecuencia & Porcentaje & $\begin{array}{l}\text { Porcentaje } \\
\text { válido }\end{array}$ & $\begin{array}{l}\text { Porcentaje } \\
\text { acumulado }\end{array}$ \\
\hline Válido & No respondió & 29 & 7,0 & 7,3 & 7,3 \\
\hline & No & 100 & 24,0 & 25,1 & 32,3 \\
\hline & Sí & 270 & 64,9 & 67,7 & 100,0 \\
\hline & Total & 399 & 95,9 & 100,0 & \\
\hline Perdidos & Sistema & 17 & 4,1 & & \\
\hline Total & & & 416 & 100,0 & \\
\hline
\end{tabular}

Fuente: población de la provincia de Santa Elena

Elaborado por: Autores.

Si la información influye en el comportamiento el 65 \% de los encuestados están conscientes e indican que si influye, el $24 \%$ expresa que no.

Tabla. No. 5 Conoce los mecanismos de verificación de la información

\begin{tabular}{|l|l|l|l|l|l|}
\hline \multicolumn{2}{|c|}{} & Frecuencia & Porcentaje & Porcentaje válido & Porcentaje acumulado \\
\hline \multirow{4}{*}{ Válido } & No respondió & 12 & 2,9 & 3,0 & 3,0 \\
\cline { 2 - 6 } & Sí & 123 & 29,6 & 30,8 & 33,8 \\
\cline { 2 - 6 } & No & 264 & 63,5 & 66,2 & 100,0 \\
\cline { 2 - 6 } & Total & 399 & 95,9 & 100,0 & \\
\hline Perdidos & Sistema & 17 & 4,1 & & \\
\hline Total & 416 & 100,0 & & \\
\hline
\end{tabular}

Fuente: población de la provincia de Santa Elena

Elaborado por: Autores

Conoce los mecanismos de verificación de la información, el 64 \% indica que no conocen los mecanismo, versus al 30 \% que indica que sí.

\section{Discusión}

Los periodistas que participaron en el grupo focal consensuaron que la inmediatez está fuertemente ligada a las redes sociales, las mismas que son revisadas de manera constante en el ordenador desde la cabina de los medios de comunicación o desde el teléfono móvil, según criterio, fortalece el trabajo periodístico, particularmente en la radio, debido a que una de las características de este medio, es la inmediatez. En este sentido Alsina (1989) manifiesta que la radio induce a las personas y proyecta sus pensamientos, del mismo modo ocurre con las redes sociales, estas inducen a la población en la formación de 
criterios, por lo que en la práctica periodística la verificación y contrastación de la información juega un rol importante frente a la inmediatez.

Los periodistas participantes identifican dos fases en el contexto de inmediatez de la información: La primera es la velocidad y cantidad de información que llega por distintos canales; mensajes de textos, SMS, Redes sociales; WhatsApp, Facebook, Instagram, estas son utilizadas por la ciudadanía, para emitir mensajes que en el contexto sociocultural a estas fuentes informativas se las asume como criterio de verdad. La segunda fase es la cronológica, es decir, el tiempo, esta fase es primordial debido a que la calidad de la información depende del tratamiento o de proceso del hecho noticioso, esto determina qué información difundir y el enfoque con el cual se emite, versus a la información mediática. De Alzaga (2012) en su libro los riesgos del periodismo en tiempo de las redes, manifiesta que existen periodistas que utilizan las redes sociales como fuente de información, esto es corroborado por los periodistas que participaron en el grupo focal, los cuales indicaron que es común la utilización de las redes para nutrir la noticia. La población encuestada manifiesta en un 71 $\%$ que recibe como verdadera la información en las redes sociales, por lo que los profesionales tienen una gran responsabilidad en la utilización las redes.

En este sentido los profesionales concuerda que en el contexto periodístico, el contenido que se difunde le da mayor valor a la inmediatez y no a la inversa, es decir, no solo por el hecho de difundir de manera inmediata, significa qué hecho o situación va a causar impacto, de esto depende el enfoque de la noticia, y si esta, está verificada. Por lo tanto, el periodista debe tener una agudeza moral para evitar caer en la inmediatez. Mientras se cumpla con el tratamiento correcto de la noticia es difícil tener inmediatez. Por otro lado, existe la responsabilidad social con los ciudadanos, de los encuestados el 64 \% dice que la información influye en el comportamiento de las personas. Los periodistas coinciden en que, si se ejecuta la recopilación de datos previo a iniciar una grabación o un reporte en vivo, pero destacan que hacerlo sin antes tener datos preliminares es un error y que por lo general se comete cuando el periodista inicia en el oficio.

La inmediatez no incide en el tratamiento del hecho noticioso, sino que la correlación es a la inversa, que la inmediatez de la información que se difunde depende del tiempo que se invierte en el riguroso proceso de tratamiento de la noticia. Existe un desgaste del periodista, al pretender responder en todas las plataformas al mismo tiempo, es lo que desmejora el tratamiento del hecho noticioso, exponen que la inmediatez es manejable dentro de los conceptos discutidos anteriormente, pero que la saturación de trabajo provoca imprecisiones, plantea que la respuesta del periodismo ante la inmediatez deben ser con datos breves, informar un hecho importante, el contraste y todo el tratamiento de la noticia. En este contexto Salavarría (2010) reconoce que el periodismo en su recolección de información tiene una ardua tarea, sobre todo, en la verificación de los hechos. 
Finalmente, dentro de la administración de las fuentes de información, la teoría explica que las voces oficiales son las más apropiadas para la construcción de una noticia (Marín, Armentía, \& Caminos, 2011), sin embargo, un hallazgo importante en la investigación revela que desde la vigencia de la Ley Orgánica de Comunicación (LOC), el periodismo abusa de la presunción, en este contexto los periodistas de Santa Elena entienden que no tiene la autoridad para juzgar, pero hablar de presunción cuando el contexto es obvio para la ciudadanía, aumentan las crítica para los medios de comunicación y a los periodistas que son tachados de amañados por intereses políticos y corporativos (Cuesta Moreno, 2012). Esto se convierte en un catalizador que le brinda credibilidad a los medios de comunicación informales, como son las redes sociales.

\section{Conclusiones}

La inmediatez no es nueva para el periodismo es un contexto de la profesión siempre se ha expuesto, sin embargo en la actualidad el escenario cambia, cuando aparece Internet y toda una gama de herramientas que permiten a los usuarios acceder a información, esta saturación provoca el desgaste y pérdida de vigencia de la información, por el desarrollo de la tecnología.

Los periodistas de Santa Elena identifican dos fases dentro de la inmediatez: Primero es la cantidad de información que llega a través de diversos canales y desde sus fuentes informativas, reporteros, ciudadanos y cibernautas. La segunda fase es el tiempo que le toma al periodista determinar qué información es de mayor utilidad para su público objetivo, además de darle el enfoque adecuado para convertirlo en noticia y llene las expectativas de las audiencias.

Estas dos fases ubican al periodista en un lugar de saturación de información, donde debe tener los cinco sentidos en alerta para no realizar emisiones imprudentes que puedan atentar contra la tranquilidad de la ciudadanía o que merme el grado de confiabilidad y credibilidad que perciben los oyentes del medio.

Estos dos actos dentro de la inmediatez dejan la responsabilidad de lo difundido sobre un ser humano que no solo está recibiendo aquella información, está locutando, filtrando información y publicando en redes sociales, esta saturación de tareas sobre los periodistas pone en riesgo la calidad de la información.

Es innegable que un recurso básico para la construcción de la noticia son las fuentes de información, que son de donde provienen los datos que llegan al periodista y que le permiten hilvanar una historia o describir un suceso.

En temas de profundidad, las fuentes de información guían el camino del periodista que está en la permanente búsqueda de la verdad.

Las fuentes de información fueron clasificadas dentro del periodismo convencional en dos grupos: las oficiales y no oficiales. El primer grupo corresponde a los familiares de algún afectado o testigos oculares; el segundo 
grupo alberga a los voceros oficiales de las instituciones públicas, policía, fiscalía, administradores de justicia, y todo aquel que represente una posición oficial ante un hecho noticioso.

Por otro lado, las redes sociales eran únicamente una esfera de interacción social, pero evolucionó de tal manera que en la actualidad es una de las fuentes primarias de información, tanto oficial como no oficial, tomando en cuenta que las instituciones públicas y privadas tienen cuentas oficiales en todas las redes sociales.

La verificación es un acto básico que corresponde realizar al periodista antes de difundir un hecho noticioso, las audiencias no se toman el tiempo para comprobar si la información que les llega en verdadera o no, tampoco ejercen algún método científico para determinar el sesgo que tiene una nota periodística, la ciudadanía no lo hace, ese es el trabajo del periodista, los oyentes únicamente toman como un hecho real y como verdad lo que escuchan o leen.

Ante los acontecimientos de inmediatez, los periodistas de Santa Elena sugieren que como tarea principal está la verificación de los hechos, es este sentido el reportero tiene un rol importante previo a comunicar el suceso. Es decir, el acto de chequear la información debe ser una acción sine qua non del periodismo de todos los tiempos.

El tratamiento del hecho noticioso en la radio se cumple por cuotas, en muchas ocasiones no todos los involucrados de un hecho se encuentran en el sitio del evento, el reportero realiza una primera entrega periodística y luego de cerrar se moviliza para ubicar a la contraparte, si hubiese un tercer protagonista, se ejecuta el mismo proceso, la dinámica del tratamiento del hecho noticioso se asemeja a la de Twitter, que provee de información breve en 180 caracteres y hay varias publicaciones sobre un hecho para contextualizar, contrastar y cumplir con todo el proceso riguroso del tratamiento del hecho noticiosos.

Finalmente, el resultado de la investigación arroja que la inmediatez, es un elemento positivo, el reto es que el profesional del periodismo pueda brindarle un buen tratamiento del hecho noticioso, estas circunstancias demandan de una mejor preparación de los periodistas.

\section{Referencias bibliográficas}

Aller, E. (2012). La Verdad Periodistica y las fuentes de información. Facultad de Periodismo y comunicación Social UNLP, 1-13.

Atamara, T. (2012). La prudencia en la inmediatez del acto informativo. Ecos de la Comunicación, 95 - 115.

Borges, R. R. (1998). La objetividad periodística, un mito persistente. Revista Latina de Comunicación Social.

Brusi, D., Alfaro, P., \& González, M. (2008). Los riesgos geológicos en los medios de comunciación. El tratamiento de la informaión de las catastrofes naturales como recurso didactico. Enseñanza de las Ciencias de la Tierra, 154-166. 
Canavilhas, J. (2012). Uso y credibilidad de fuentes periodísticas 2.o. El profesional de la información, 63-69.

Canavilhas, J. (2012). Uso y credibilidad de fuentes periodísticas 2.o. El profesional de la información. elprofesionaldelainformacion.com/contenidos/2012/enero/o8.pdf, 63-69., 63-69.

Castillo, M. J. (22 de Marzo de 2017). El problema de las falsedades en las redes sociales. Obtenido de https://idus.us.es/handle/11441/70265?: https://idus.us.es/handle/11441/70265?

Cornella, A. (2000). Cómo sobrevivir a la infoxicación. 8.

Cuesta Moreno, Ó. J. (2012). Los axiomas de la radio, tecnología y periodismo radiofónico. Red de Revistas Científicas de América Latina, el Caribe, España y Portugal.

De Alzaga, P., Romero, P., Llop, P., Tascón, M., Ribas, C., Rodríguez, J., \& Tudela Flores, A. (2012). Los riesgos del periodismo en tiempos de redes. Madrid: Evoca Comunicación e Imagen.

De la Cruz Conforme, V. (7 de agosto de 2017). Grupo focal. (J. D. Laínez, Entrevistador)

Del Peso Arias, P. (7 de agosto de 2017). Grupo focal. (J. D. Laínez, Entrevistador)

Eco, U. (2016). Guerra y web 2.o, decepción y esperanza frente a los medios digitales del siclo XXI. Univesidad los Andes, 98-110.

Eco, U. (2016). Guerra y Web 2.0, descripción y esperanza frente a los medios digitales. Universidad los Andes, 98-110.

Enriquez , Juan Gabriel; Casas , Sandra Isabel;. (2013). Usabilidad en aplicaciones móviles. ICT-UNPA, 62.

Flora Marín, José Ignacio Armentia, José Caminos. (2011). El tratamiento informativo de las víctimas de violencia de género en Euskadi: Deia. Comunicación y sociedad, 435 -466.

García, S. (2010). Crisis del periodismo de fuentes. Las prácticas del periodismo en España en el accidente de Spanair. Revista Latina de Comunicación Social, 516-537.

Hamui Sutton, A., \& Varela Ruiz, M. (2012). Técnica de grupos focales. Investigacion en educacion Médica.

Hernandez Sampieri, R. (2016). Metodología de la Investigación. Mexico D.F.: Interamericana editores, S.A. DE C.V.

Jima Romero, C. (2013). Utilización de las redes sociales en el contexto del periodismo deportivo radial. Tesis de pregrado. Cuenca, Ecuador: Universidad de Cuenca.

Lino De la Cruz, J. (7 de agosto de 2017). Grupo focal. (J. Drouet Laínez, Entrevistador)

Marcos Recio, J. C., Sánchez Vigil, J. M., \& Serrada Gutiérrez, M. (2009). Nuevos paradigmas periodisticos y documentales en los periódicos digitales: Estudio de casos en España. Investigación Bibliotecnológica, 43-65.

Marín, F., Armentía, J., \& Caminos, J. (2011). el tratamiento informativo de las víctimas de violencia de género en Euskadi: Deia, El Correo, El País y Gara (2002-2009). Comunicación y sociedad, 435-466.

Mauricio Aguilar-Garavito, Wilson Ramírez. (2015). Estructura y Contenidos Básicos para El Programa De Monitoreo. En M. Aguilar Garavito, \& W. Ramirez, Monitoreo a procesos de restauración ecológica (pág. 255). Bogotá, Colombia: Alexander Von Humboldt.

Monfort, N. (mayo de 2013). http://hdl.handle.net/10234/66678. Obtenido de http://www. adcomunicarevista.com/ojs/index.php/adcomunica/article/view/118/149: http://repositori.uji.es/xmlui/handle/10234/66678

Najarro, L. (2011). Noticiero radiofónico: un generador de imágenes e imaginación. Cuba.

Penalva, C. (2002). El tratamiento de la violencia en los medios de comunicación. Alternativas. Cuadernos de Trabajo Social, 395-412. 
Potter, D. (2006). Manual de periodismo independiente. Estados Unidos: Mildred Solá Neely. Rojas, A., \& R, T. (2012 ). La prudencia en la inmediatez del acto informativo . Ecos de la Comunicación, 95-111.

Rosique-Cedillo, G., \& Barranquero-Carretero, A. (2015). Periodismo lento (slow journalism) en la era de la inmediatez. Experiencias en Iberoamérica. researchgate.net, 451-462.

Ruiz, F. J. (2014). Cómo entender al periodismo : selección de la obra de Wolfgang Donsbach. Argentina : Minigraf SRL.

Salavarría, R. (2010). Estructura de la Convergencia. En S. Ramón, Estructura de la Convergencia (págs. 27-40). España, Santiago de Compostela : Editorial de la Universidad Santiago de Compostela . 
